\title{
Distribución de las concentraciones de glucosa e insulina basal, HOMA IR y HOMA ßcell en niños y adolescentes de la ciudad de Maracaibo, Venezuela
}

\author{
Luz Marina Moralesa, Xiomara Raleigh ${ }^{b}$, Virginia Fernández, \\ Emperatriz Molero-Conejo.
}

Distribution of fasting glucose, insulin, homeostasis model assessment (HOMA) insulin resistance (IR) and HOMA B cell in children and adolescents from Maracaibo, Venezuela

Background: The raising prevalence of obesity among children increases the risk of insulin resistance and its adverse metabolic consequences. Aim: To determine the distributions of fasting serum glucose, insulin, HOMA IR and HOMA ß cell in a representative sample of children and adolescents from Maracaibo-Venezuela. Subjects and Methods: Fasting insulin and glucose were measured in 418 children and adolescents (191 boys and 227 girls) of 7, 9, 11, 13, 15 years of age. HOMA IR and HOMA ß cell were calculated. Results: Insulin levels were lower in 7 and 9 year-old girls and 7 year-old boys compared with 11, 13 and 15 year-old girls and boys. Fasting glucose concentrations were similar in boys and girls. HOMA IR was lower in 7 year-old girls compared to 11,13 and 15 years-old girls, whereas boys in every age showed similar values. HOMA $\lesssim$ cell was higher in 11 and 13 year-old girls. Conclusions: Our findings provide useful values to assess insulin resistance and ß-cell functioning in children and adolescents (Rev Méd Chile 2007; 135: 205-11).

(Key w ords: Beta cells, pancreatic; Homeostasis; Insulin resistance; Obesity)

Recibido el 28 de febrero, 2006. Aceptado el 15 de junio, 2006.

Sección Bioquímica, Instituto de Investigaciones Clínicas, Facultad de Medicina, Universidad del Zulia. Maracaibo, Venezuela.

aLicenciada en Nutrición y Dietética. MgSc en Bioquímica

bTSU en Informática

Correspondencia a: Luz Marina Morales G. Instituto de Investigaciones Clínicas «Dr. Américo Negrete», Facultad de Medicina, La Universidad del Zulia. Apartado 23, Maracaibo-Venezuela. Fax: 0261-7597247. E mail: lucecita24@yahoo.com. vfernandez@luz.edu.ve 
$\mathrm{L}$ as alteraciones del metabolismo de la glucosa e insulina, asociadas con dislipidemias e hipertensión se han relacionado con riesgo para el desarrollo de enfermedad cardiovascular y diabetes tipo $2^{1,2}$. Se ha encontrado que este conjunto de variables de riesgo persisten desde la niñez hasta la fase adulta3,4. Además, la insulinorresistencia y la hiperinsulinemia constituyen factores de riesgo del síndrome metabólico en la población puberal ${ }^{5}$.

Actualmente, se ha planteado la necesidad de realizar prevención de los factores de riesgos de enfermedad cardiovascular en niños y adolescentes. Esto está relacionado con el hecho de que la ateroesclerosis comienza en etapas tempranas de la vida y los hábitos que se han asociado con mayor riesgo para los desórdenes ateroescleróticos se establecen al final de la adolescencia ${ }^{6}$. En las últimas dos décadas, se ha observado un incremento en la prevalencia de obesidad en niños y adolescentes ${ }^{7,8}$, aumentando su riesgo para alteraciones metabólicas. Las concentraciones de insulina basal se han considerado como un medidor seguro para la determinación de insulinorresistencia en los estudios epidemiológicos, debido a que existe una buena comelación entre ésta y los resultados reportados empleando clamp hiperinsulinémicos euglicémicos ${ }^{9}$. Haffner y col $^{10}$ propusieron un modelo homeostático para evaluar insulinomesistencia (HOMA IR) y función de las células B (HOMA ßcell) en estudios epidemiológicos en adultos, considerando los niveles basales de insulina y glucosa. Se han realizado pocos estudios a nivel nacional para determinar la utilidad del HOMA en la evaluación de alteraciones metabólicas en niños y adolescentes, que permita identificar el riesgo de estos grupos para enfermedad cardiovascular y diabetes. Este estudio tuvo como objetivo determinar la distribución de las concentraciones de glucosa en ayunas, insulina basal, HOMA IR y HOMA ßcell en niños y adolescentes de la ciudad de Maracaibo, Venezuela, con la finalidad de establecer las asociaciones entre los niveles de insulina y HOMA IR que permitan el diagnóstico de insulinorresistencia en los niños y adolescentes de nuestra región.

\section{PACIENTES Y MÉTODO}

Se estudiaron 418 niños y adolescentes (191 varones y 227 mujeres) de 7, 9, 11, 13, y 15 años que asistieron a planteles oficiales y privados, pertenecientes a los estratos II, III, IV, y V de acuerdo al método Graffar modificado. Para obtener la muestra en los 13 planteles educativos seleccionados de las diferentes parroquias del municipio de Maracaibo, se elaboraron las listas de todos los alumnos con las edades de interés.

Los niños y adolescentes recibieron información verbal y escrita del propósito del estudio y su participación fue voluntaria, con aprobación de su representante. El estudio fue aprobado por el comité de ética del Instituto de Investigaciones Clínicas Dr. Américo Negrette de la Facultad de Medicina de la Universidad de Zulia (L.U.Z.).

A cada niño se le realizaron las mediciones antropométricas de talla, peso, pliegues subcutáneos, circunferencia de brazo izquierdo, se calculó la edad cronológica y se clasificó de acuerdo a los indicadores de dimensiones corporales: PesoEdad, Talla-Edad y Peso-Talla según gráficos de la OMS y su interpretación combinada. Además, se clasificó por el índice de masa corporal $\left(\mathrm{P} / \mathrm{T}^{2}\right)$. Se emplearon los valores límites para diagnóstico de desnutrición y obesidad (un valor menor al percentil 10 o mayor al percentil 90) elaborados por Hernández-Valera y $\operatorname{col}^{11}$, y los indicadores de composición corporal: pliegues cutáneos (tricipital en $\mathrm{mm}$ ), área muscular y área grasa ${ }^{12}$. Aplicando estos criterios, se seleccionaron los niños y adolescentes con estado nutricional normal. Los niños que se encontraron con desnutrición (48 niños y adolescentes) y con obesidad (60 niños y adolescentes) fueron excluidos.

La evaluación de los caracteres sexuales: glándula mamaria, genitales y vello pubiano, se hizo siguiendo los estadios de maduración de Tanner ${ }^{13}$.

A cada niño y adolescente, después de $12 \mathrm{~h}$ de ayuno, se le midieron en sangre venosa los niveles séricos de glicemia, por el método enzimático de glucosa oxidasa (Glucosa liquicolor, GODPAP Method, Human) y niveles de insulina por radioinmunoensayo (Coat-A-Count Insulin, Diagnostic Products Corporation).

Se calculó HOMA IR y HOMA ßcell empleando las fórmulas de Mattews y col${ }^{14}$.

Estadística. Se aplicó la prueba de normalidad de Kolmogorov-Smirnov a los valores de insulina, la cual determinó que no seguían la distribución 
gaussiana y se procedió a la normalización de la muestra mediante la transformación logarítmica (logaritmo decimal) de estos valores.

Los valores se representan como media \pm desviación estándar. Se realizó el análisis de ANOVA con postest de Bonfferoni, utilizando el programa estadístico computarizado (SPSS). Se consideró una $\mathrm{p}<0,05$ como significativo.

\section{Resultados}

Al realizar la evaluación nutricional, se encontraron 307 niños y adolescentes con estado nutricional normal (165 niñas y 142 varones), distribuidas en las diferentes edades estudiadas. De acuerdo a la escala de Tanner para determinar la maduración sexual, las niñas y varones de 7 años se ubicaron en Tanner 1, las niñas y los varones de 9 años en Tanner 1-2, estando el mayor porcentaje de los varones en Tanner 1, las niñas de 11 años en Tanner 2-3, mientras que los varones en Tanner 12, las niñas de 13 años en Tanner 3-4 y los varones en Tanner 2-3, las niñas de 15 años en Tanner 4-5 y los varones en Tanner 3-4.

En la Tabla 1 se muestran las medias y los percentiles de las concentraciones de insulina clasifi- cados por edad y sexo. Las niñas de 7 y 9 años y los varones de 7 años presentaron niveles de insulina basal significativamente menores ( $p<0,001$ ), comparados con las niñas y varones de 11, 13, y 15 años. La media de insulina basal de los varones de 9 años solamente fue significativamente menor ( $p \varangle 0,001$ ) cuando se comparó con los de 11 años. Tanto en las niñas como en los varones de 11, 13 y 15 años no se observaron diferencias significativas en los niveles basales de insulina. En el percentil 75, las niñas de 13 años presentaron los niveles más altos de insulina, mientras que en los varones, el valor más elevado se observó en los de 15 años. Aun cuando no se observaron diferencias significativas, los varones de 9, 11, 13 y 15 años presentaron niveles de insulina inferiores a los de las niñas de su misma edad.

Las medias de las concentraciones de glucosa en ayuno fueron similares, tanto en niñas como en varones, en las diferentes edades estudiadas. Esto mismo fue observado en el percentil 75 (Tabla 2).

Las niñas de 7 años presentaron valores de HOMA IR significativamente menores $(p<0,05)$ comparados con las niñas de 11, 13, 15. La niñas de 9 años tuvieron valores de HOMA IR significativamente menores $(p<0,01)$ que las niñas de 11 y 13 años, pero similares a las de 15 años. En los

Tabla 1. Percentiles de las concentraciones de insulina en niños y adolescentes

\begin{tabular}{|c|c|c|c|c|c|c|}
\hline \multirow{2}{*}{$\begin{array}{l}\text { Sexo/ } \\
\text { Edad (años) }\end{array}$} & \multirow{2}{*}{ Medias (IC 95\%) } & \multicolumn{5}{|c|}{ Percentiles } \\
\hline & & 5 & 25 & 50 & 75 & 95 \\
\hline \multicolumn{7}{|l|}{ Hembras } \\
\hline 7 (29) & $3,52(2,83-4,39)^{\mathrm{a}}$ & 1,24 & 2,49 & 3,40 & 4,92 & 13,46 \\
\hline $9(26)$ & $4,95(4,02-6,11)^{b}$ & 1,73 & 3,37 & 5,19 & 7,13 & 10,58 \\
\hline $11(46)$ & $8,91(7,59-10,44)^{\mathrm{C}}$ & 2,40 & 6,77 & 9,95 & 13,15 & 17,62 \\
\hline $13(37)$ & $8,96(7,21-11,13)^{\mathrm{C}}$ & 2,78 & 5,75 & 8,50 & 14,39 & 27,20 \\
\hline $15(27)$ & $8,09(6,43-10,17)^{\mathrm{c}}$ & 1,99 & 6,00 & 8,20 & 12,19 & 20,18 \\
\hline \multicolumn{7}{|l|}{ Varones } \\
\hline $7(19)$ & $3,86(2,61-5,69)^{\mathrm{a}}$ & 1,19 & 1,99 & 3,69 & 6,89 & 31,09 \\
\hline $9(39)$ & $4,72(3,86-5,79)^{\mathrm{a}}$ & 1,40 & 3,40 & 5,20 & 6,69 & 16,0 \\
\hline $11(22)$ & $6,44(4,72-8,79)^{b}$ & 1,23 & 4,59 & 6,60 & 9,10 & 21,72 \\
\hline $13(39)$ & $5,96(4,72-7,54)^{b}$ & 1,40 & 4,39 & 5,90 & 8,60 & 21,69 \\
\hline $15(23)$ & $6,17(4,75-8,01)^{b}$ & 1,74 & 3,90 & 6,30 & 10,20 & 15,59 \\
\hline
\end{tabular}

Concentraciones de insulina: $\mu \mathrm{U} / \mathrm{ml}$.

Letras diferentes indican diferencias significativas entre las diferentes edades, con $p<0,001$.

Entre paréntesis el número de observaciones. 
Tabla 2. Percentiles de las concentraciones de glucosa en ayunas en niños y adolescentes

\begin{tabular}{|lcccccc|}
\hline $\begin{array}{l}\text { Sexo/ } \\
\text { Edad (años) }\end{array}$ & Medias (IC 95\%) & \multicolumn{5}{c}{ Percentiles } \\
& & 5 & 25 & 50 & 75 & 95 \\
\hline Hembras & & & & & & \\
$7(29)$ & $78,66(75,6-81,7)$ & 62,50 & 73,50 & 80,00 & 84,00 & 92,00 \\
$9(26)$ & $79,08(75,4-82,8)$ & 63,35 & 73,00 & 79,00 & 83,25 & 103,70 \\
$11(46)$ & $80,93(78,0-83,9)$ & 65,35 & 73,75 & 81,00 & 88,00 & 100,65 \\
$13(37)$ & $76,59(62,2-80,9)$ & 47,30 & 70,50 & 81,00 & 86,00 & 92,40 \\
$15(27)$ & $81,19(76,8-85,6)$ & 60,00 & 75,00 & 82,00 & 89,00 & 103,00 \\
Varnes & & & & & & \\
$7(19)$ & $83,42(78,6-88,3)$ & 72,00 & 76,00 & 80,00 & 88,00 & 112,00 \\
$9(39)$ & $81,79(78,5-85,1)$ & 69,00 & 76,00 & 80,00 & 88,00 & 108,00 \\
$11(22)$ & $83,86(79,7-88,0)$ & 67,30 & 76,75 & 85,50 & 88,50 & 102,70 \\
$13(39)$ & $77,56(73,6-81,5)$ & 55,00 & 70,00 & 76,00 & 84,00 & 104,00 \\
$15(23)$ & $81,19(74,9-82,1)$ & 60,00 & 75,00 & 82,00 & 89,00 & 103,00 \\
\hline
\end{tabular}

Concentraciones de glucosa: $\mathrm{mg} / \mathrm{dl}$.

Entre paréntesis el número de observaciones.

varones no hubo diferencia significativa en el HOMA-IR entre los diferentes grupos etarios. En el percentil 75, las niñas de 11 años presentaron el valor más alto de HOMA IR. En este percentil, en los varones el valor más alto de HOMA IR se encontró en los de 15 años (Tabla 3).
Las niñas de 11, 13 y 15 años tuvieron una secreción de insulina más elevada ( $p \varangle 0,001$ ), medida por HOMA ßcell, cuando se compararon con las niñas de 7, 9 años. En los varones no se observaron diferencias significativas en ningún grupo etario. Las niñas de 13 años tuvieron valores de

Tabla 3. Percentiles de H O M A-IR en niños y adolescentes

\begin{tabular}{|lcccccc|}
\hline $\begin{array}{l}\text { Sexo/ } \\
\text { Edad (años) }\end{array}$ & Medias (IC 95\%) & \multicolumn{5}{c|}{ Percentiles } \\
& & 5 & 25 & 50 & 75 & 95 \\
\hline Niñas & & & & & & \\
$7(29)$ & $0,81(0,6-1,1)^{\mathrm{a}}$ & 0,22 & 0,47 & 0,67 & 0,93 & 3,02 \\
$9(26)$ & $1,09(0,9-1,3)^{\mathrm{a}}$ & 0,32 & 0,65 & 1,06 & 1,46 & 2,08 \\
$11(46)$ & $2,01(1,7-2,3)^{\mathrm{b}}$ & 0,47 & 1,37 & 1,94 & 2,67 & 3,97 \\
$13(37)$ & $2,19(1,6-2,8)^{\mathrm{b}}$ & 0,40 & 1,07 & 1,61 & 2,59 & 5,90 \\
$15(27)$ & $1,92(1,5-2,4)^{\mathrm{b}}$ & 0,32 & 1,25 & 1,60 & 2,36 & 4,72 \\
Varones & & & & & & \\
$7(19)$ & $1,24(0,4-2,1)^{\mathrm{a}}$ & 0,23 & 0,39 & 0,83 & 1,38 & 8,59 \\
$9(39)$ & $1,15(0,9-1,4)^{\mathrm{a}}$ & 0,30 & 0,59 & 1,11 & 1,35 & 3,11 \\
$11(22)$ & $1,64(1,1-2,2)^{\mathrm{a}}$ & 0,24 & 1,07 & 1,41 & 1,71 & 5,42 \\
$13(39)$ & $1,52(1,0-2,0)^{\mathrm{a}}$ & 0,26 & 0,73 & 1,18 & 1,65 & 4,87 \\
$15(23)$ & $1,41(1,1-1,2)^{\mathrm{a}}$ & 0,33 & 0,71 & 1,18 & 2,02 & 3,13 \\
\hline
\end{tabular}

Letras diferentes indican diferencias significativas entre las diferentes edades, con $p<0,001$.

Entre paréntesis el número de observaciones. 
HOMA ßcell significativamente mayores ( $p<0,01$ ) que los varones de su misma edad. En el percentil 75 las niñas y los varones de 13 años presentaron el mayor valor de HOMA ßcell (Tabla 4).

En cada grupo etario, tanto en niñas como en varones, se observó correlación altamente significativa $(r=0,98, p>0,0001)$ entre $\log _{e}$ insulina $y$ $\log _{\mathrm{e}}$ HOMA IR.

\section{DisCUSIÓN}

Este estudio presenta la distribución por percentiles de los niveles basales de insulina, glucosa, HOMA IR y HOMA ßcell en una muestra representativa de niños y adolescentes del área de Maracaibo, Estado Zulia. En nuestra región no existe un consenso acerca de los puntos de corte que permitan definir hiperinsulinemia o un insulinorresistencia medida por HOMA IR. Dado que la hiperinulinemia e insulinorresistencia aumentan el riesgo para diabetes ${ }^{15}$ y enfermedad cardiovascular, la definición en forma precisa de estas variables permitirá la identificación de niños y adolescentes en riesgo y aplicar medidas preventivas a temprana edad.
El incremento de las concentraciones de insulina, de HOMA IR y HOMA ßcell en las niñas de 11 y 13 comparadas con las de 7 y 9 años, puede estar relacionado con una disminución de la sensibilidad de la insulina en el inicio de la pubertad, sin la presencia de alteraciones en la función de las células $\beta$. En los varones no se observaron diferencias entre los diferentes grupos etarios. Según esto, pareciera que las niñas son más insulinorresistentes que los varones, similar a lo reportado por Morán y $\mathrm{col}^{16}$ en un estudio realizado en niños entre 10-14 años, sometidos a clamp euglicémico. Ellos encontraron un incremento de la insulino resistencia en el inicio de la pubertad (Tanner 2), retornando a los niveles de la fase prepuberal al final de la pubertad (Tanner 5). El pico ocurrió en Tanner 3, en ambos sexos. En nuestro estudio, esto último se observó en las niñas pero no en los varones. Según nuestros datos, no se encontraron diferencias entre varones y niñas en ninguna de las variables estudiadas, similar a lo reportado por Viso y cols ${ }^{17}$, quienes no encontraron diferencias significativas entre género en los niveles de insulina en niños eutróficos y obesos. A diferencia de lo comunicado por otros autores ${ }^{18,19}$, quienes encontraron

Tabla 4. Percentiles de H O M A-ßcell en niños y adolescentes

\begin{tabular}{|lrrrrrr|}
\hline Sexo/ & & & \multicolumn{5}{c|}{ Percentiles } \\
Edad (años) & Medias (IC 95\%) & & & & \\
& & 5 & 25 & 50 & 75 & 95 \\
\hline Niñas & & & & & & \\
$7(29)$ & $90,13(66,9-113,4)^{\mathrm{a}}$ & 17,32 & 49,25 & 72,28 & 132,32 & 201,33 \\
$9(26)$ & $124,91(98,1-151,7)^{\mathrm{a}}$ & 43,31 & 68,58 & 116,24 & 178,46 & 270,29 \\
$11(46)$ & $220,40(168,6-262,2)^{\mathrm{b}}$ & 53,62 & 125,83 & 164,17 & 257,43 & 683,63 \\
$13(37)$ & $238,74(182,6-294,9)^{\mathrm{b}}$ & 78,67 & 148,06 & 180,77 & 301,92 & 638,90 \\
$15(27)$ & $193,92(139,3-248,6)^{\mathrm{b}}$ & 76,03 & 98,20 & 148,10 & 277,02 & 535,47 \\
Varones & & & & & & \\
$7(19)$ & $103,42(58,6-148,2)^{\mathrm{a}}$ & 22,98 & 42,54 & 65,82 & 137,39 & 342,25 \\
$9(39)$ & $130,45(96,6-164,3)^{\mathrm{a}}$ & 24,41 & 53,47 & 96,50 & 188,68 & 367,64 \\
$11(22)$ & $170,71(93,7-247,7)^{\mathrm{a}}$ & 23,91 & 56,67 & 100,14 & 213,89 & 665,86 \\
$13(39)$ & $170,29(120,6-220,0)^{\mathrm{a}}$ & 36,30 & 81,49 & 130,87 & 265,74 & 510,94 \\
$15(23)$ & $174,35(124,2-224,5)^{\mathrm{a}}$ & 45,44 & 85,50 & 127,74 & 244,14 & 387,55 \\
\hline
\end{tabular}

Letras diferentes indican diferencias significativas entre las diferentes edades, con $p<0,001$. Entre paréntesis el número de observaciones. 
diferencias en la sensibilidad a la insulina entre niñas y varones. Se puede pensar que existe una tendencia central en cada una de las variables estudiadas, más que un efecto del género en los extremos. Esto último es importante debido a que son los valores extremos los que se consideran para clasificar los individuos.

Se encontró una correlación significativa entre las concentraciones de insulina y HOMA IR, lo cual era lo esperado, debido a que los valores de HOMA-IR se derivan de las concentraciones de insulina y glucosa. Asimismo, debido a que las concentraciones de insulina y HOMA IR siguieron la misma distribución, se puede considerar a este último como un medidor tan bueno como la insulina basal para establecer insulino resistencia. Keskin y $\mathrm{col}^{20}{ }^{2}$, demostraron que el HOMA-IR tuvo una alta especificidad y sensibilidad para la medición de insulinorresistencia en niños y adolescentes. Esto mismo es lo que ha sido reportado en adultos, en quienes HOMA IR y HOMA ßcell se han considerado como un modelo útil para evaluar insulinorresistencia y función de la célula $ß$ en estudios epidemiológicos, en los cuales sólo cuenta con muestras basales ${ }^{10,21}$. Además, Keskin y $\operatorname{col}^{20}$ establecieron que el HOMA IR era más aceptable que la relación glucosa/insulina en ayunas y el índice de cuantificación de la sensibilidad a la insulina (QUICKI).

\section{REFERENCIAS}

1. LakKa HM, LaAksonen DE, LakKa TA, Niskanen LK, Kumpusalo E, Tuomilehto J et aL. The metabolic syndrome and total and cardiovascular disease mortality in middle-aged men. JAMA 2002; 288: 2709-16.

2. Haffner SM, Valdez RA, Hazuda HP, Mitchell BD, Morales PA, Stern MP. Prospective analysis of the insulin-resistance syndrome (syndrome $\mathrm{X}$ ). Diabetes 1992; 41: 715-22.

3. Bao W, Srinivasan SR, Wattigney WA, Berenson GS. Persistence of multiple cardiovascular risk clustering related to syndrome $\mathrm{X}$ from childhood to young adulthood: the Bogalusa Heart Study. Arch Intern Med 1994; 154: 1842-7.
En todas las edades estudiadas no se observaron diferencias significativas en las concentraciones de glucosa entre las niñas y varones, similar a lo reportado por Burritt y $\mathrm{Col}^{22}$ quienes no encontraron diferencias significativas entre edades o género en las concentraciones séricas de glucosa. Allard y col ${ }^{24}$ comunicaron niveles de glucosa superiores a los encontrados en este grupo de niños y adolescentes. En nuestro estudio, los valores de glucosa en el percentil 75 estuvieron entre 83 y $89 \mathrm{mg} / \mathrm{dl}$, los comunicados por Allard y col${ }^{23}(5,0-5,3 \mathrm{mmol} / \mathrm{L}=90$ $96 \mathrm{mg} / \mathrm{dL}$ ), corresponden al percentil 95 de nuestros niños y adolescentes en los diferentes grupos etarios. De acuerdo a lo presentado en la Tabla 2, si tomamos el percentil 25 y 75 como límites para los niveles de glucosa en los niños y adolescentes entre 7-15 años, éstos se encontranían entre 70 y $89 \mathrm{mg} / \mathrm{dl}$. Guillaume y $\mathrm{col}^{19}$ y Chen y $\mathrm{col}^{24}$, han reportando valores similares a los encontrados en este estudio.

En conclusión, los niveles basales de insulina se asociaron con los valores de HOMA IR en el grupo de niños y adolescentes estudiados, siendo las concentraciones de insulina basal un marcador tan bueno como el HOMA IR para la determinación de insulinorresistencia en estos grupos etarios. Los resultados de este estudio proporcionan valores que permitirán evaluar los estados de insulinorresistencia y funcionamiento de las células B en niños y adolescentes.

4. Chen W, Srinivasan SR, L S,$X_{u}$ J, Berenson GS. Metabolic syndrome variables at low levels in childhood are beneficially associated with adulthood cardiovascular risk: the Bogalusa Heart Study. Diabetes Care 2005; 28: 126-31.

5. BuczkowsKa O. Insulin resistance and hiperinsulinemia-risks factors of the syndrome in pubertal population. Endokrynol diabetol Chor Przemiany Materii Wieku Rozw 2005; 11: 109-14.

6. American Academy of Pediatrics. Committee on Nutrition. Cholesterol in Childhood. Pediatrics 1998; 101: 141-7.

7. Troiano RP, Flegal KM. Overweight children and adolescents: description, epidemiology, and demographics. Pediatrics 1998; 101: 497-504. 
8. Tremblay MS, WiLms JD. Secular trends in the body mass index of Canadian children. CMAJ 2000; 163: 1429-33.

9. LAAKSO M. How good a marker is insulin level for insulin resistance? Am J Epidemiol 1993; 137: 959-65.

10. HafFner SM, Miettinen H, Stern M. The Homeostasis Model in the San Antonio Heart Study. Diabetes Care 1997; 20: 1087-92.

11. Hernández-Valera Y, Henríquez PG, Arenas O, García Blanco M, Cardena Y. Índice de masa corporal P/T2. Valores para diagnóstico de desnutrición en niños venezolanos de 2 a 10 años de edad. XXV Jornadas Nac Ped Puer 1986. En: Manual de crecimiento y desarrollo. Caracas, FUNDACREDESA, 1997; $186 \mathrm{p}$.

12. FRISANCHO AR. New norms of upper limb fat and muscle areas for assessment of nutritional status. Am J Clin Nutr 1981; 34: 2540-5.

13. TANNER JM. Growth at adolescence. $2^{\text {nd }}$ ed Oxford: Blackwell Scientific Publications; 1962.

14. Matthews DR, Hosker JP, Rudenski AS, Naylor BA, TREACHER DF, TuRNER RC. Homeostasis model assessment: insulin resistance and beta cell function from fasting plasma glucose and insulin concentrations in man. Diabetología 1985; 28: 412-9.

15. Rosenberg B, Morán A, Sinaiko AR. Insulin resistance (metabolic) syndrome in children. Panminerva Med 2005; 47: 229-44.

16. Morán A, Jacobs DR Jr, Steinberger J, Hong CP, PRINEAS R, LUEPKER R ET AL. Insulin resistance during puberty: results from clamp studies in 357 children. Diabetes 1999; 48: 2039-44.

17. Viso G, Solano L, Sánchez A, Portilo Z, Llovera D. Insulina sérica en niños y adolescentes obesos y eutróficos. An Venez Nutr 2004; 17: 57-63.

18. Rönnemaa T, Knip M, Lautala P, Viikari J, Uhari M, LeINo A ET AL. Serum insulin and other cardiovas- cular risk indicators in children, adolescents and young adults. Ann Med 1991; 23: 67-72.

19. Guilaume M, Lapidus L, Beckers $F$, Lambert A, BJORNTORP P. Cardiovascular risk factors in children from the Belgian province of Luxembourg. The Belgian Luxembourg Child Study. Am J Epidemiol 1996; 144: 867-80.

20. Keskin M, Kurtoglu S, Kendirci M, Atabek ME, YAZICI C. Homeostasis model assessment is more reliable than the fasting glucose/insulin ratio and quantitative insulin sensitivity check index for assessing insulin resistance among obese children and adolescents. Pediatrics 2005; 115: e500503.

21. Yeni-Komshian H, Carantoni M, Abbasi F, Reaven GM. Relationship between several surrogate estimates of insulin resistance and quantification of insulin-mediated glucose disposal in 490 healthy nondiabetic volunteers. Diabetes Care 2000; 23: 171-5.

22. Burritt MF, SLockbower JM, Forsman RW, Oxford KP, Bergstrall EJ, Smith WA. Pediatric reference intervals for 19 biologic variables in healthy children. Mayo Clin Proc 1990; 65: 329-36.

23. Allard P, Delvin EE, Paradis G, Hanley JA, O'Loughun J, Lavalée C ET aL. Distribution of fasting plasma insulin, free fatty acids, and glucose concentrations and of Homeostasis Model Assessment of insulin resistance in a representative sample of Quebec children and adolescents. Clin Chem 2003; 49: 644-9.

24. Chen W, SRinivasan SR, Elikasabany A, Berenson GS. Cardiovascular risk factors clustering features of insulin resistance syndrome (Syndrome $\mathrm{X}$ ) in biracial (Black-White) population of children, adolescents, and young adults: the Bogalusa Heart Study. Am J Epidemiol 1999; 150: 667-74.

Agradecimiento

Agradecemos a la Corporación del Desarrollo de la Región Zuliana (CORPOZULA) por su apoyo en la recolección de datos. 